\title{
Liquid-phase aerobic oxidation of benzyl alcohol catalyzed by mechanochemically synthesized manganese oxide
}

\author{
ILYAS Mohammad $^{1 *}$, SIDDIQUE Mohsin $^{1} \&$ SAEED Muhammad ${ }^{1,2}$ \\ ${ }^{1}$ National Centre of Excellence in Physical Chemistry, University of Peshawar, KPK 2500, Pakistan; \\ ${ }^{2}$ Department of Chemistry, Government College University, Faisalabad, Pakistan
}

Received June 25, 2012; accepted October 8, 2012; published online May 20, 2013

\begin{abstract}
Benzyl alcohol oxidation by molecular oxygen using solid manganese oxide as a catalyst and $n$-heptane as the solvent was studied in the liquid phase. The catalyst was synthesized by a simple mechanochemical process at room temperature and was characterized by different physical methods. The catalyst was highly active and $100 \%$ selective for benzyl alcohol conversion to benzaldehyde. The solid catalyst can be recovered from the reaction mixture by simple filtration and can be re-used. We found $n$-heptane to be an excellent solvent for the oxidation reaction, and that the catalyst did not leach into solution.
\end{abstract}

benzaldehyde, catalyst selectivity, chemical analysis, $n$-heptane, environment

Citation: Ilyas M, Siddique M, Saeed M. Liquid-phase aerobic oxidation of benzyl alcohol catalyzed by mechanochemically synthesized manganese oxide. Chin Sci Bull, 2013, 58: 2354-2359, doi: 10.1007/s11434-013-5833-z

Catalytic oxidation of alcohols to the corresponding carbonyl compounds is of great importance to the scientific community and to industry throughout the world because these carbonyl compounds serve as versatile intermediates for the synthesis of many chemicals [1,2]. Conventional inorganic oxidants such as permanganates and dichromates, however, are dangerous and create a great quantity of waste [3-5]. Recovery and disposal of this waste tends to increase the number of steps in the overall chemical cycle.

Therefore, there is an emerging need for such catalytic systems to use greener oxidants such as molecular oxygen and to employ low temperatures and pressures that are close to ambient conditions. The relevant literature holds a number of reports that list molecular oxygen as the primary oxidant [6-9], while water is considered to be the best solvent [10]. However, our previous studies have shown that the use of water leads to overoxidation of alcohols to produce carboxylic acids rather than aldehydes. In such cases, the use of dry conditions with organic solvents is the best choice.

*Corresponding author (email: mohilyas52@yahoo.com)
Manganese oxide, a nonprecious metal oxide, has been used as an oxidation catalyst in several chemical processes. The catalytic properties of manganese oxide depend on the preparation conditions, the surface structure, oxidation state, surface area, size distribution and the morphology of the particles. Many synthetic routes have been used to prepare manganese oxide, including the sol-gel route, pulsed laser deposition, gel hydrothermal oxidation, oxidation-reduction, homogenous precipitation, cobalt salt decomposition, a staged oxidation process, and a mechanochemical process. The mechanochemical process is suitable for large-scale synthesis because of its simplicity and low cost [11]; manganese oxide can be obtained by solid-state displacement reaction, either by simple milling or by milling followed by heat treatment.

The main objective of the present work was to investigate the catalytic activity of manganese oxide, prepared using the solid-state mechanochemical process, for the oxidation of primary alcohols. For this study, we chose the conversion of benzyl alcohol to benzaldehyde and benzoic acid as the model oxidation reaction. 


\section{Experimental}

\subsection{Materials}

Benzyl alcohol (Scharlau, Spain), $n$-heptane (Acros, USA), manganese chloride (Acros), and potassium permanganate (Merck, Germany) were used as received. Molecular oxygen and nitrogen were provided by BOC Pakistan and were further purified by passing through standard traps (CRS) to remove trace amounts of water and oils. Specific oxygen traps (CRS) were used to remove any oxygen present in the nitrogen.

\subsection{Catalyst preparation}

The required quantity of solid $\mathrm{Mn}$ (II) salt (i.e., manganese nitrate or manganese chloride) was mixed and ground with the desired amount of solid potassium permanganate salt in a fixed ratio of 2:3 using a mortar and pestle. The procedure was conducted in a fume cupboard at room temperature until the color of the mixture become homogeneous. The grayish black semisolid was then heated at $100^{\circ} \mathrm{C}$ for a few hours to complete the reaction between the components and a solid mass was formed. The solid was washed extensively with distilled water and then dried in a desiccator at $80^{\circ} \mathrm{C}$ for $24 \mathrm{~h}$. The final product was a black powder of manganese oxide [11]. This manganese oxide was calcined at $300^{\circ} \mathrm{C}$ in a Nabertherm programmable furnace that used a temperature rise of $5^{\circ} \mathrm{C} / \mathrm{min}$; the temperature was held at $300^{\circ} \mathrm{C}$ for $2 \mathrm{~h}$.

\subsection{Characterization}

Grain size distributions were determined for wet sample dispersions using an Analysette 22 compact particle sizer (Fritsch, Germany). BET and surface area analysis of the catalyst was conducted using a NOVA 1200e surface area and pore size analyzer (Quantachrome). Before analysis, the catalyst was degassed at $383 \mathrm{~K}$ for $2 \mathrm{~h}$ to remove volatiles that were adsorbed onto the catalyst surface. X-Ray diffraction (XRD) patterns were determined using a JEOL JDX-3532 X-ray diffractometer (JEOL, Japan). Data were acquired using $\mathrm{Cu} \mathrm{K} \alpha$ radiation with a tube voltage of 40 $\mathrm{kV}$ at $20 \mathrm{~mA}$, with a $2 \theta$ range of $0^{\circ}-70^{\circ}$. FT-IR spectra were recorded on $\mathrm{KBr}$ disks using an IR Prestige 21 spectrophotometer (Shimadzu, Japan) with a wavenumber range of $400-4000 \mathrm{~cm}^{-1}$.

\subsection{Oxidation protocol}

Oxidation reactions of benzyl alcohol in the liquid phase were carried out in a 50-mL three-neck round-bottom flask with magnetic stirring. The flask was fitted with a reflux condenser and a thermometer to determine the reaction temperature. Heating for the reaction was provided by a hot plate. Benzyl alcohol ( $2 \mathrm{mmol})$ in $10 \mathrm{~mL}$ of $n$-heptane was added to the reaction flask. After reaching the required temperature $(343 \mathrm{~K}), 0.1 \mathrm{~g}$ of catalyst was added to the flask. Oxygen was passed through the reaction mixture at a flow rate $60 \mathrm{~mL} / \mathrm{min}$, while the mixture was stirred with an agitation speed of $950 \mathrm{r} / \mathrm{min}$.

Any moisture in the supplied oxygen was removed by moisture traps. We have noted previously that the selectivity of reaction products can change if the oxygen contains moisture; we found that undried oxygen allows the formation of benzoic acid together with benzaldehyde, even at the beginning of the reaction. In the case of dry conditions, only benzaldehyde was detected as the reaction product. Before entering the reactor, the oxygen was saturated with $n$-heptane by passing it through a saturator containing solvent at the reaction temperature. This saturation of oxygen with solvent minimizes the loss of solvent with flow of oxygen from the reaction mixture. The catalyst was separated from the reaction mixture with a Whatman Glass microfiber filter using a glass syringe.

For reaction at various partial pressures of oxygen, nitrogen was admixed with oxygen. The total flow rate for these experiments was also maintained at $60 \mathrm{~mL} / \mathrm{min}$.

Analysis of the reaction mixture was conducted by GC (Clarus 500, Perkin Elmer, USA) equipped with FID. Benzyl alcohol, benzaldehyde, and benzoic acid were easily separated by on an Elite-5 GC column (N9316076, Perkin Elmer). UV-visible spectrophotometry (UV-160A, Shimadzu) was also used for benzaldehyde estimation and comparison of the results.

\section{Results and discussion}

\subsection{Particle size distribution}

The particle size of the prepared catalyst was in the range of 3-10 $\mu \mathrm{m}$.

\subsection{Surface area and pore size analysis}

The nitrogen adsorption isotherm for the catalyst was obtained by plotting the volume of nitrogen adsorbed against the relative pressure $\left(P / P_{0}\right)$. The BET surface area was found to be $72.5 \mathrm{~m}^{2} / \mathrm{g}$, while $\mathrm{BJH}$ pore radius and pore volume was determined to be $45.0 \AA$ and $0.266 \mathrm{~cm}^{3} / \mathrm{g}$ respectively.

\subsection{XRD analysis}

The XRD patterns of different manganese oxides catalysts are shown in Figure 1. In the XRD pattern of the prepared catalyst, peaks at $2 \theta=12.7^{\circ}, 25.2^{\circ}, 35.1^{\circ}, 40.7^{\circ}$, and $58.85^{\circ}$ are apparent and show the crystalline nature of the catalyst. Among the peaks, those at $2 \theta=12.7^{\circ}$ and $25.2^{\circ}$ are assigned to $\mathrm{s}-\mathrm{MnO}_{2}$. These peaks are very weak, suggesting that this phase is not present in our catalyst in significant 


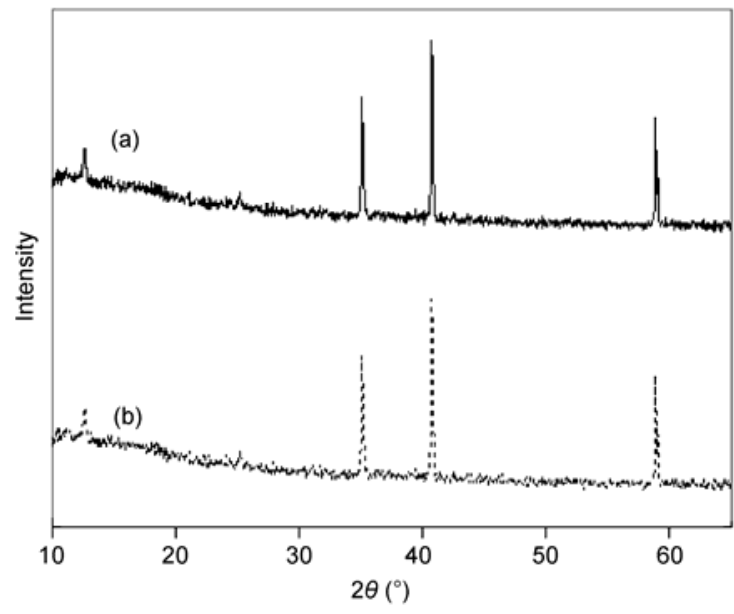

Figure 1 XRD of fresh (a) and used (b) catalysts.

quantity. The peaks at $2 \theta=35.1^{\circ}, 40.7^{\circ}$, and $58.9^{\circ}$ are assigned to $\mathrm{MnO}$ [12]. In comparing the XRD data of the prepared catalyst with reported data, it was concluded that the catalyst is a mixture of $\mathrm{MnO}$ in nature. Based on the XRD data, it was concluded that the catalytic activities of the manganese oxide are due to the presence of hydrated $\mathrm{MnO}$. The crystal size of the catalyst used for oxidation of benzyl alcohol was calculated using Scherer's equation [13] and was found to be $3.84 \mathrm{~nm}$.

\subsection{FT-IR spectra}

Figure 2 shows the FT-IR spectra of different catalysts. Absorption bands are observed in the ranges 3300-3500, $1500-1700,1000-1100$, and $500-600 \mathrm{~cm}^{-1}$. The 3300-3500 $\mathrm{cm}^{-1}$ bands are assigned to $\mathrm{O}-\mathrm{H}$ stretching vibrations; the bands observed between 1100 and $1600 \mathrm{~cm}^{-1}$ are most usually assigned to $\mathrm{O}-\mathrm{H}$ bending vibrations for groups joined to manganese. Similarly, the bands in region 500-900 $\mathrm{cm}^{-1}$ are assigned to $\mathrm{Mn}-\mathrm{O}$ vibrations. The IR spectrum suggests the existence of some interstitial bound water in the catalyst. The spectrum of the fresh catalyst suggests that it is mainly composed of $\mathrm{MnO}$, having characteristic bands at 833, 948,

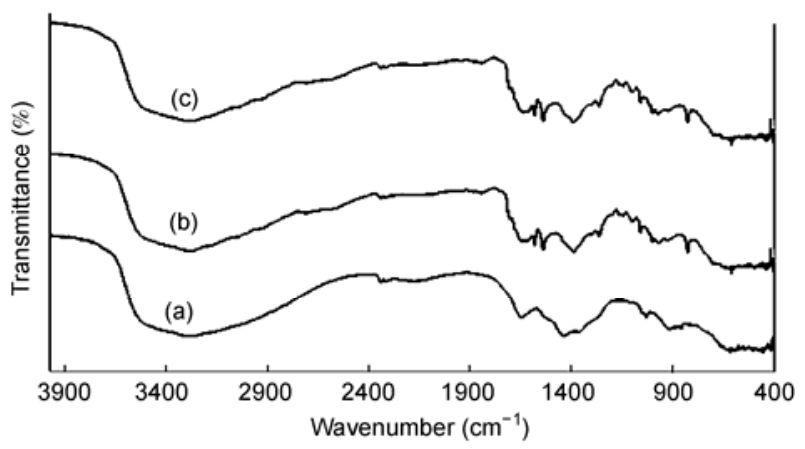

Figure 2 FT-IR spectra of fresh (a), once-used (b) and twice-used (c) catalysts. and $816 \mathrm{~cm}^{-1}$. The broad peak at $3200-3500 \mathrm{~cm}^{-1}$ was assigned to the stretching vibration of $\mathrm{H}_{2} \mathrm{O}$ and the hydroxyl group in the lattice. The peak near $1600 \mathrm{~cm}^{-1}$ in the fresh catalyst (Figure 2(a)) was assigned to the bending vibration of water and $\mathrm{OH}$, which suggests that hydroxyl group is present in the prepared catalyst. The spectra of used catalysts (Figure 2(b) and (c)) show bands at $1592 \mathrm{~cm}^{-1}$, which may be due to $\mathrm{MnH}_{2}$ with $\mathrm{H}$ derived from benzyl alcohol [14-21].

\subsection{Scanning electron microscopy}

A scanning electron micrograph (SEM) of the catalyst is shown in Figure 3. Similar morphology has been observed for plate-like layered manganese oxide catalysts [22-24]; however, the larger surface area in these studies was probably caused by the presence of pillared material (e.g., alumina, lithium). The presence of pillared material creates a larger interlayer distance and results in pores having the form of parallel plates.

Figure 3 suggests that the particle size of the catalyst is of the order of a micron, while XRD data suggests particle size of the order of a nanometer. The average crystallite size is calculated by Scherrer equation based on XRD data, while the SEM image shows the size of particles that are aggregates of several crystallites.

\subsection{Leaching of the catalyst}

Catalyst leaching is known to be a problem in liquid-phase catalytic reactions. We performed a simple experiment to determine whether catalyst leaching occurred in our system. $n$-Heptane was stirred with catalyst in a flask under the same experimental conditions $\left(70^{\circ} \mathrm{C}\right.$ for $2 \mathrm{~h}$, constant oxygen flow). After $2 \mathrm{~h}$, the catalyst was removed from the reaction mixture and the benzyl alcohol substrate was added. The reaction mixture was stirred for a further $2 \mathrm{~h}$, after which no oxidation products were detected by GC (FID) or UV-visible spectrophotometry. This confirmed the absence of leaching of the catalyst.

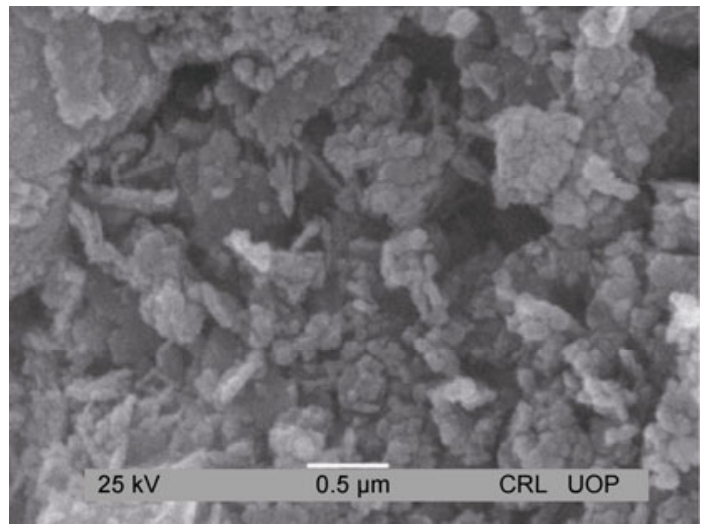

Figure 3 SEM image of prepared catalyst. 


\subsection{Catalyst loading}

The effect of catalyst load on the progress of the benzyl alcohol oxidation was monitored over the range 0.03-0.12 g, keeping all other parameters of temperature and pressure constant. Figure 4 shows that the conversion of benzyl alcohol increases with catalyst loading, although the increase is nonlinear. Full conversion $(100 \%)$ was achieved at loadings of $0.15 \mathrm{~g}$ and higher.

\subsection{Life span of catalyst}

To investigate catalyst reuse, the used catalyst was collected from the reaction and was washed several times in an ethanol-water mixture. The washed catalyst was then reused. We found that used $\mathrm{MnO}$ had the same catalytic performance as fresh catalyst, as shown in Figure 5. These results confirm the high effectiveness of the current process for easy and convenient oxidation of benzyl alcohol by an easily removed and reusable manganese catalyst.

\subsection{Time profile investigation}

The use of manganese oxide as catalyst resulted in gradual

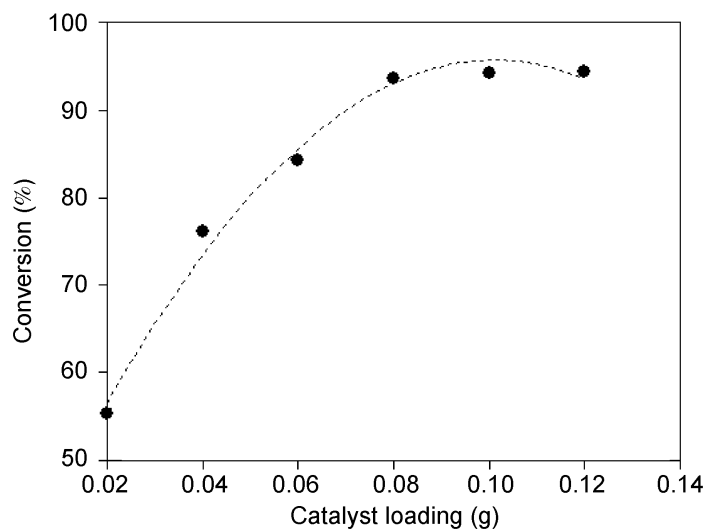

Figure 4 Effect of catalyst loading on oxidation of benzyl alcohol. Initial concentration of benzyl alcohol: $0.2 \mathrm{~mol} / \mathrm{L}$ in $n$ - heptane, volume: $10 \mathrm{~mL}$, temperature $343 \mathrm{~K}$, oxygen pressure $1 \mathrm{~atm}\left(1 \mathrm{~atm}=1.013 \times 10^{5} \mathrm{~Pa}\right), 950$ $\mathrm{r} / \mathrm{min}, 90 \mathrm{~min}$.

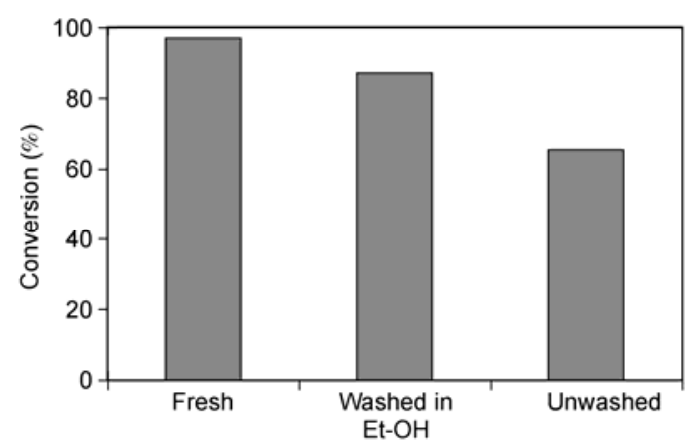

Figure 5 Life span of catalyst for the oxidation of benzyl alcohol. Initial concentration of benzyl alcohol: $0.2 \mathrm{~mol} / \mathrm{L}$ in $n$-heptane, volume: $10 \mathrm{~mL}$, temperature $343 \mathrm{~K}$, oxygen pressure $1 \mathrm{~atm}, 950 \mathrm{r} / \mathrm{min}, 90 \mathrm{~min}$. conversion of benzyl alcohol to benzaldehyde over time. The time profile investigation of benzyl alcohol oxidation was monitored continuously. The time profile study was carried out at $343 \mathrm{~K}$. In the early part of the reaction, only benzaldehyde was detected as the reaction product. As the conversion of benzyl alcohol to benzaldehyde neared completion ( $95 \%)$, formation of benzoic acid was detected. This shows the consecutive nature of the reaction. Figure 6 shows the time profile of the reaction. Formation of benzoic acid was only detected at the beginning of the oxidation process when oxygen was not dried by passing it through a silica moisture trap. We concluded that dry conditions are necessary to control the selectivity of the product distribution. Figure 7 shows the time profile for benzyl alcohol oxidation at various temperatures in range $313-343 \mathrm{~K}$, with all other experiment conditions remaining unchanged.

\subsection{Effect of oxygen partial pressure}

The effect of oxygen partial pressure on benzyl alcohol oxidation was investigated over the range 33-101 kPa with a constant initial concentration of $0.2 \mathrm{~mol} / \mathrm{L}$ benzyl alcohol at $70^{\circ} \mathrm{C}$. The results in Figure 8 show that the conversion of benzyl alcohol increases with increasing oxygen partial

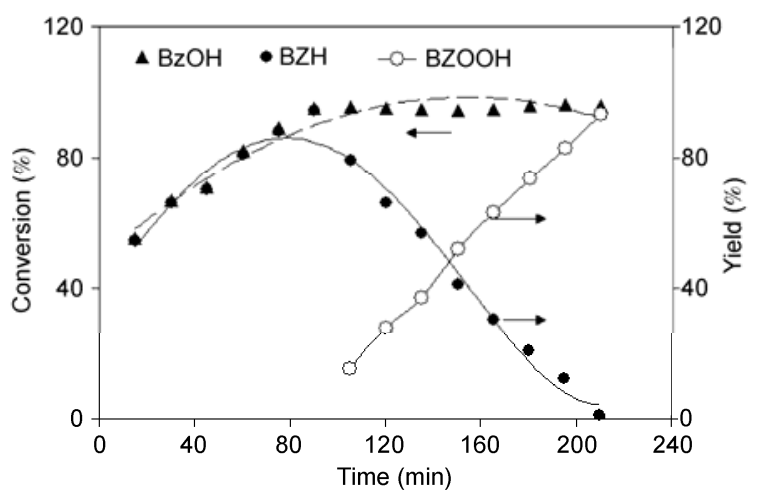

Figure 6 Time profile for conversion of benzyl alcohol (BzOH) to benzaldehyde $(\mathrm{BzH})$ and benzoic acid $(\mathrm{BzOOH})$ at $343 \mathrm{~K}$. Initial concentration of benzyl alcohol: $0.2 \mathrm{~mol} / \mathrm{L}$ in $n$-heptane, volume: $10 \mathrm{~mL}$, oxygen pressure $1 \mathrm{~atm}, 950 \mathrm{r} / \mathrm{min}, 90 \mathrm{~min}$.

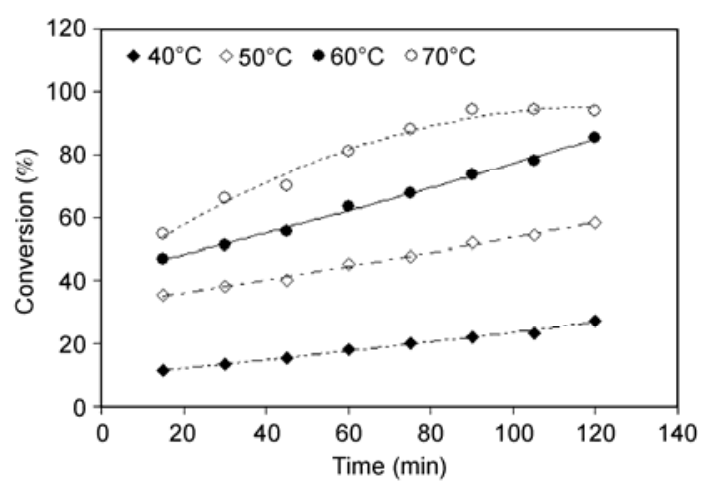

Figure 7 Effect of temperature on the conversion benzyl alcohol. Initial concentration of benzyl alcohol: $0.2 \mathrm{~mol} / \mathrm{L}$ in $n$-heptane, volume: $10 \mathrm{~mL}$, oxygen pressure $1 \mathrm{~atm}, 950 \mathrm{r} / \mathrm{min}, 90 \mathrm{~min}$. 
Table 1 Comparison of catalysts: Liquid-phase oxidation of benzyl alcohol using molecular oxygen as oxidant catalyzed by manganese-containing catalysts

\begin{tabular}{lcccccc}
\hline \multicolumn{1}{c}{ Catalyst } & Amount $(\mathrm{mg})$ & $\mathrm{BzOH}(\mathrm{mmol})$ & Solvent $($ volume, $\mathrm{mL})$ & Temperature $(\mathrm{K})$ & Conversion $(\%)$ & References \\
\hline $\mathrm{MnO}_{x}(110)$ & 50 & 1 & Toluene $(10)$ & 383 & 72 & {$[16]$} \\
$\mathrm{K}(2) \mathrm{Mn}(1) / \mathrm{C}$ & 200 & 2 & Toluene $(10)$ & 373 & 92 & {$[25]$} \\
$\mathrm{K}(1) \mathrm{Mn}(1) / \gamma \mathrm{Al}_{2} \mathrm{O}_{3}$ & 200 & 2 & Toluene (10) & 373 & 42 & {$[26]$} \\
$\mathrm{Mn}_{x} \mathrm{O}_{y}$ & 100 & 2 & $n$-Heptane (10) & 343 & 100 & Present work \\
\hline
\end{tabular}

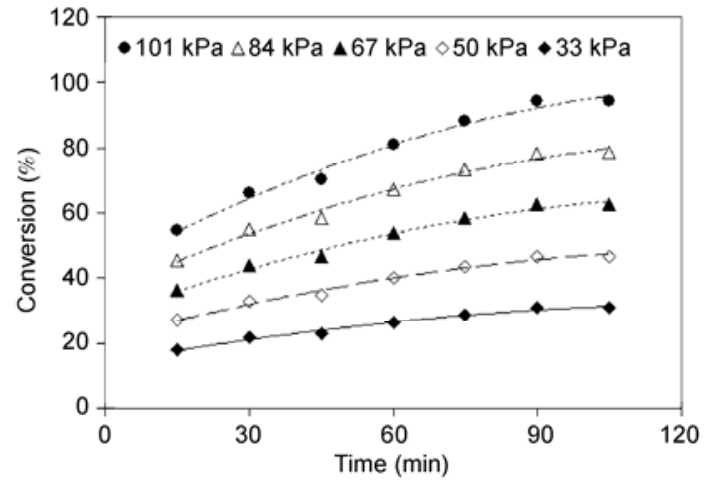

Figure 8 Effect of partial pressure of oxygen on the reaction of benzyl alcohol at $343 \mathrm{~K}$. Initial concentration of benzyl alcohol: $0.2 \mathrm{~mol} / \mathrm{L}$ in $n$ heptane, volume: $10 \mathrm{~mL}, 950 \mathrm{r} / \mathrm{min}, 90 \mathrm{~min}$.

pressures.

The conversion of benzyl alcohol was reduced from $96 \%$ to $23 \%$ when the partial pressure of oxygen was reduced from 101 to $34 \mathrm{kPa}$. The low conversion in the presence of nitrogen could be due to oxygen from the lattice taking part in the reaction [18]. This apparent reduction of manganese oxide could be limited to a few surface layers because no difference in the XRD patterns of the unused and used catalyst was observed (Figure 2).

\subsection{Comparison of laboratory-prepared and commercial manganese oxide}

The activity of our laboratory-prepared catalyst was compared with commercially available manganese oxide catalyst. The results show that our laboratory-prepared catalyst was superior to the commercial catalyst (Figure 9).

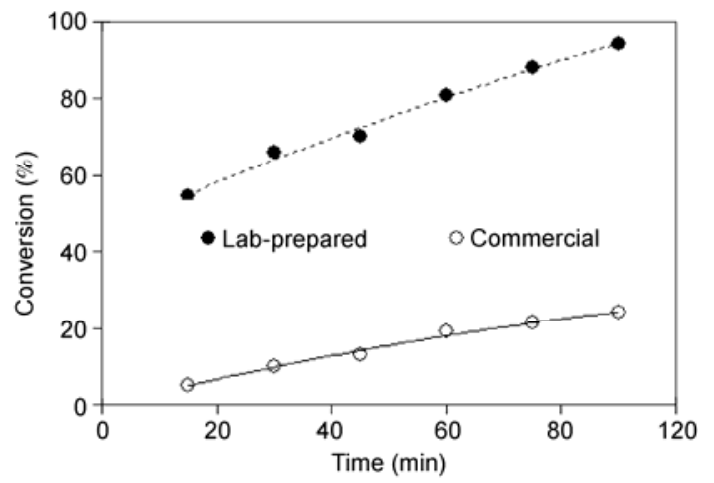

Figure 9 Comparison of laboratory-prepared and commercial catalyst. Initial concentration of benzyl alcohol: $0.2 \mathrm{~mol} / \mathrm{L}$ in $n$-heptane, volume: $10 \mathrm{~mL}$, temperature $343 \mathrm{~K}$, oxygen pressure $1 \mathrm{~atm}, 950 \mathrm{r} / \mathrm{min}, 90 \mathrm{~min}$.
Table 2 Comparison of catalyst activity between batches

\begin{tabular}{ccc}
\hline Batch number & Conversion $(\%)$ & Time $(\min )$ \\
\hline 1 & 96 & 90 \\
2 & 97 & 90 \\
3 & 96 & 90 \\
\hline
\end{tabular}

\subsection{Comparison with other catalysts}

Comparison of the activity of the present catalyst with other manganese-containing catalysts (Table 1) shows that the present catalyst is highly active and superior for benzyl alcohol conversion to benzaldehyde.

\subsection{Batch analysis}

To determine the reproducibility of our catalyst preparation, three batches of catalyst were prepared and tested for activity in benzyl alcohol oxidation. The results shown in Table 2 show no significant difference between batches.

\section{Conclusions}

Manganese oxide was prepared by a simple mechanochemical process in the solid state and was used as catalyst for the oxidation of benzyl alcohol. The synthesized manganese oxide was characterized as having an octahedral layer structure. The catalyzed oxidation of benzyl alcohol was carried out in $n$-heptane solvent over a temperature range 313-343 K and with the partial pressure of oxygen ranging between 34 and $101 \mathrm{kPa}$. The prepared catalyst showed remarkable catalytic activity for oxidation of benzyl alcohol under relatively mild conditions. The prepared manganese oxide was found to be an effective, reusable, and easily separated heterogeneous catalyst for oxidation of benzyl alcohol.

The authors thank the Higher Education Commission of Pakistan for financial assistance under the Indigenous PhD Fellowship Program.

1 Velu S, Wang L, Okazaki M, et al. Characterization of MCM-41 mesoporous molecular sieves containing copper and zinc and their catalytic performance in the selective oxidation of alcohols to aldehydes. Microp Mesop Mat, 2002, 54: 113-126

2 Besson M, Gallezot P. Selective oxidation of alcohols and aldehydes on metal catalysts. Catal Today, 2000, 57: 127-141

3 Tembe S, Patrick G, Scurrell M. Acetic acid production by selective 
oxidation of ethanol using Au catalysts supported on various metal oxide. Gold Bull, 2009, 42: 321-327

4 Manosroi J, Manosroi A, Sripalakit P. Extraction of solasodine from dry fruits and leaves of solanum laciniatum ait. And the synthesis of 16-dehydropregnenolone acetate from solasodine by phase-transfer catalysis. In: Brovelli E, Chansakaow S, Farias D, et al., eds. Proceedings of the WOCMAP III-Thailand Congress on Medicinal and Aromatic Plants, Chiang Mai, Thailand, 2005, 679

5 Sheldon R A, Arends I, Hanefeld U. Green Chemistry and Catalysis. Weinheirri: Wiley-VCH, 2007

6 Premalatha K, Raghavan P, Viswanathan B. Liquid phase oxidation of benzyl alcohol with molecular oxygen catalyzed by metal chromites. Appl Catal A: General, 2012, 419-420: 203-209

7 Esteruelas M A, García-Obregón T, Herrero J, et al. Osmiumcatalyzed oxidation of primary alcohols with molecular oxygen. Organometallics, 2011, 30: 6402-6407

8 Shi Z, Zhang C, Tang C, et al. Recent advances in transition-metal catalyzed reactions using molecular oxygen as the oxidant. Chem Soc Rev, 2012, 41: 3381-3430

9 Takagaki A, Tsuji A, Nishimura S, et al. Genesis of catalytically active gold nanoparticles supported on hydrotalcite for base-free selective oxidation of glycerol in water with molecular oxygen. Chem Lett, 2011, 40: 150-152

10 Frassoldati A, Pinel C, Besson M. Promoting effect of water for aliphatic primary and secondary alcohol oxidation over platinum catalysts in dioxane/aqueous solution media. Catal Today, 2011, 173: 81-88

11 Li Q, Luo G, Li J, et al. Preparation of ultrafine $\mathrm{MnO}_{2}$ powders by the solid state method reaction of $\mathrm{KMnO}_{4}$ with $\mathrm{Mn}$ (II) salts at room temperature. J Mat Proc Technol, 2003, 137: 25-29

12 Wang L C, He L, Liu Y M, et al. Effect of pretreatment atmosphere on co oxidation over $\alpha-\mathrm{Mn}_{2} \mathrm{O}_{3}$ supported gold catalysts. J Catal, 2009, 264: $145-153$

13 Ilyas M. Oxidation of benzyl alcohol in liquid phase catalyzed by oxides of nickel. J Chem Soc Pakistan, 2010, 31: 526

14 Yuan C, Gao B, Su L, et al. Interface synthesis of mesoporous $\mathrm{MnO}_{2}$ and its electrochemical capacitive behaviors. J Colloid Interf Sci,
2008, 322: 545-550

15 Gandhe A R, Rebello J S, Figueiredo J L, et al. Manganese oxide OMS-2 as an effective catalyst for total oxidation of ethyl acetate. Appl Catal B: Environ, 2007, 72: 129-135

$16 \mathrm{Hu} \mathrm{J}$, Sun K, He D, et al. Amorphous manganese oxide for catalytic aerobic oxidation of benzyl alcohol. Chin J Catal, 2007, 28: 1025-1027

17 Kannan R, Jegan A, Ramasubbu A, et al. Synthesis and catalytic studies of layered and oms type nano manganese oxide material. Dig J Nanomat Biostruct, 2011, 6: 755-760

18 Makwana V D, Son Y C, Howell A R, et al. The role of lattice oxygen in selective benzyl alcohol oxidation using OMS-2 catalyst: A kinetic and isotope-labeling study. J Catal, 2002, 210: 46-52

19 Trawczyński J, Bielak B, Miśta W. Oxidation of ethanol over supported manganese catalysts-Effect of the carrier. Appl Catal B: Environ, 2005, 55: 277-285

20 Zaki M I, Hasan M A, Pasupulety L, et al. Co and ch4 total oxidation over manganese oxide supported on $\mathrm{ZrO}_{2}, \mathrm{TiO}_{2}, \mathrm{TiO}_{2}-\mathrm{Al}_{2} \mathrm{O}_{3}$ and $\mathrm{SiO}_{2}-\mathrm{Al}_{2} \mathrm{O}_{3}$ catalysts. New J Chem, 1999, 23: 1197-1202

21 Doheim M, Hanafy S, El-Shobaky G. Catalytic conversion of ethanol and isopropanol ver the $\mathrm{Mn}_{2} \mathrm{O}_{3} / \mathrm{Al}_{2} \mathrm{O}_{3}$ system doped with $\mathrm{Na}_{2} \mathrm{O}$. Mater Lett, 2002, 55: 304-311

22 Zhang X, Ji L, Zhang S, et al. Synthesis of a novel polyanilineintercalated layered manganese oxide nanocomposite as electrode material for electrochemical capacitor. J Power Sourc, 2007, 173: 1017-1023

23 Nakayama M, Konishi S, Tagashira H, et al. Electrochemical synthesis of layered manganese oxides intercalated with tetraalkylammonium ions. Langmuir, 2005, 21: 354-359

24 Yang X, Makita Y, Liu Z H, et al. Novel synthesis of layered graphite oxide-birnessite manganese oxide nanocomposite. Chem Mat, 2003, 15: 1228-1231

25 Tang Q, Liu T, Yang Y. Role of potassium in the aerobic oxidation of aromatic alcohols over $\mathrm{K}^{+}$-promoted $\mathrm{Mn} / \mathrm{C}$ catalysts. Catal Commun, 2008, 9: 2570-2573

26 Tang Q, Huang X, Wu C, et al. Structure and catalytic properties of K-doped manganese oxide supported on alumina. J Mol Catal A: Chem, 2009, 306: 48-53

Open Access This article is distributed under the terms of the Creative Commons Attribution License which permits any use, distribution, and reproduction in any medium, provided the original author(s) and source are credited. 